\title{
Small dispersion limit of the Korteweg-deVries equation with periodic initial conditions and analytical description of the Zabusky-Kruskal experiment
}

\author{
Guo Deng ${ }^{\mathrm{a}}$, Gino Biondini ${ }^{\mathrm{a}, \mathrm{b}}$, Stefano Trillo $^{\mathrm{c}}$ \\ ${ }^{a}$ State University of New York at Buffalo, Department of Physics, Buffalo, NY 14260-2900 \\ ${ }^{b}$ State University of New York at Buffalo, Department of Mathematics, Buffalo, NY 14260-2900 \\ ${ }^{c}$ Department of Engineering, University of Ferrara, Via Saragat 1, 44122 Ferrara, Italy
}

\begin{abstract}
We study the small dispersion limit of the Korteweg-de Vries (KdV) equation with periodic boundary conditions and we apply the results to the Zabusky-Kruskal experiment. In particular, we employ a WKB approximation for the solution of the scattering problem for the $\mathrm{KdV}$ equation [i.e., the time-independent Schrödinger equation] to obtain an asymptotic expression for the trace of the monodromy matrix and thereby of the spectrum of the problem. We then perform a detailed analysis of the structure of said spectrum (i.e., band widths, gap widths and relative band widths) as a function of the dispersion smallness parameter $\epsilon$. We then formulate explicit approximations for the number of solitons and corresponding soliton amplitudes as a function of $\epsilon$. Finally, by performing an appropriate rescaling, we compare our results to those in the famous Zabusky and Kruskal's paper, showing very good agreement with the numerical results.
\end{abstract}

Keywords: Korteweg-de Vries equation, inverse scattering, WKB method, solitons.

\section{Introduction}

In their seminal work in 1965 [33], Zabusky and Kruskal (ZK) performed numerical experiments on the KortewegdeVries $(\mathrm{KdV})$ equation with small dispersion,

$$
u_{t}+6 u u_{x}+\epsilon^{2} u_{x x x}=0
$$

[where subscripts $x$ and $t$ denote partial differentiation and $\epsilon>0$ is a small dispersion parameter], which they had obtained as a long-wave approximation of the Fermi-Pasta-Ulam oscillator chain. They numerically integrated (1.1) with a cosine initial condition, in an attempt to understand the thermalization process. As is now well known, however, they observed recurrences of the initial state as shown in Fig. 1 (right). Zabusky and Kruskal characterized this behavior in terms of the elastic interactions of particle-like entities which they termed "solitons". Two years later, Gardner, Green, Kruskal and Miura [6] developed an analytical method, subsequently called the inverse scattering

Email addresses: guodeng@buffalo.edu (Guo Deng), biondini@buffalo.edu (Gino Biondini), stefano.trillo@unife.it (Stefano Trillo) 
[Figures were moved to the end of the manuscript in compliance with submission instructions.]

Figure 1. Left: The eight solitons emerging from the Zabusky-Kruskal experiment; from Ref. [33]. Right: Contour plot of the solution of the Zabusky-Kruskal experiment. The vertical axis is space, and the horizontal axis is time. Note how only eight peaks are visible in the left figure, whereas at least ten excitations can be clearly identified in the right figure.

transform (IST) [1, 17], to solve the initial value problem for the KdV equation with localized initial conditions. The IST was also extended to the case of periodic boundary conditions $[1,11,16,5,17]$. Other works also showed that (1.1) possesses an infinite number of conserved quantities and is an infinite-dimensional, completely integrable system. Those results led to the development of the theory of integrable nonlinear wave equations, which continues to this day.

The ZK recurrences are usually explained in terms of soliton interactions. It has been recently shown, however, that this is not the case [9]. Since the KdV equation is amenable to analytical treatment, one can think of characterizing the emergence of solitons in the Zabusky-Kruskal (ZK) using the IST. However, it is not possible to do so using the IST for the infinite line, since no recurrence exists in this case. Rather, one must make use of the IST for the periodic case. Indeed, the ZK results were characterized from a spectral point of view in [19] by numerically integrating the scattering problem for the $\mathrm{KdV}$ equation. The number of solitons and the soliton amplitudes of the ZK problem were also discussed in [19], and those results were recently revisited in [3], which also addressed previous issues arising in the direct numerical integration of the $\mathrm{KdV}$ equation [24, 25, 26]. No analytical description was present in either of those works, however. Theoretical results about the zero dispersion limit of the Korteweg-deVries equation with periodic initial data were obtained in [28] using Lax-Levermore theory [12]. But, surprisingly, an analytical treatment of the ZK experiment has not been given in the literature to the best of our knowledge.

The goal of this work is remedy the above-mentioned deficiency and provide a full, analytical characterization of the ZK experiment. Recall that the Lax pair of (1.1) is given by

$$
\begin{gathered}
\epsilon^{2} v_{x x}+(u(x, t)+\lambda) v=0, \\
v_{t}=\left(u_{x}+\mu\right) v+(4 \lambda-2 u) v_{x} .
\end{gathered}
$$

where (1.2) [which is the time-independent Schrödinger equation] is referred to as the scattering problem and (1.3) as the time evolution. Therefore, to accomplish our goal, we need to study the small-dispersion (or semiclassical) limit of the spectral problem (1.2). Since the spectrum of (1.2) is independent of time, in what follows we can take $t=0$. Recall also that the Schrödinger equation with a periodic potential is called Hill's equation, and the special case of a cosine potential is referred to as Mathieu's equation.

Since no closed-form expression is known for the solutions of Mathieu's equation, to study the small-dispersion limit we use the Wentzel-Kramers-Brillouin (WKB) method. A first step in the application of the WKB method to the study of Mathieu's equation in the semiclassical limit was done in [22], in which a simple, qualitative study of the asymptotic limit was given. Note however that, due to the different scalings considered, even those qualitative results would not be readily applicable for our purposes. Moreover, the treatment was brief (only the eikonal equation was used), and no study was done of the number and size of bands and gaps as a function of $\epsilon$, which is necessary in order to characterize the $\mathrm{ZK}$ experiment. Here we obviate this problem and we derive a complete description of the small-dispersion limit of the KdV equation with single-lobe, periodic potential, which we then apply specifically to the Zabusky-Kruskal experiment. In particular, our approach allows to characterize analytically the two distinctive traits of the problem, namely, the fact that, as $\epsilon \rightarrow 0$ : (i) the number of bands grows proportionally to $1 / \epsilon$, and (ii) the relative width of a growing fraction of them shrink below a critical value, which defines the "soliton-like" excitations of the problem. In particular, these results imply that, as $\epsilon \rightarrow 0$, the ZK problem gives rise to a "soliton gas".

The outline of this work is the following: In section 2, the WKB approximation for Mathieu's equation is presented and the trace of the monodromy matrix is obtained. The corresponding analysis of the spectrum is presented in section 3. The results from WKB are compared with the ZK numerical experiment in section 5. Section 6 discusses how the results generalize in a straightforward way to single-lobe, periodic initial conditions. Section 7 concludes the work with some final remarks. 


\section{WKB analysis for Mathieu's equation}

Motivated by the Zabusky-Kruskal experiment, we consider the small-dispersion limit of the Schrödinger equation with a cosine potential, i.e., we rewrite (1.2) as

$$
\epsilon^{2} \phi_{x x}+Q(x) \phi=0
$$

where

$$
Q(x)=\lambda-\cos x .
$$

Note that changing $\cos x$ into $-\cos x$ has no effect on the analysis thanks to the translation invariance of the $\mathrm{KdV}$ equation.

Let us briefly recall essential concepts from Floquet theory, referring the reader to $[8,14]$ for further details. If $y(x)$ solves (2.1), $y(x+2 \pi)$ does as well. Given two linearly independent solutions $y_{1}(x)$ and $y_{2}(x)$, one can introduce the fundamental matrix solution

$$
Y(x)=\left(\begin{array}{ll}
y_{1} & y_{2} \\
y_{1}^{\prime} & y_{2}^{\prime}
\end{array}\right)
$$

of the first-order system associated with (2.1). Of course, any other fundamental matrix solution $\tilde{Y}(x)$ of the system is obtained from it as $\tilde{Y}(x)=Y(x) Z$, with $Z$ being a constant invertible matrix. Also, $Y(x+2 \pi)$ is also a fundamental matrix solution, since $Q(x+2 \pi)=Q(x)$. Thus, we can write $Y(x+2 \pi)=Y(x) M \forall x \in \mathbb{R}$, where the monodromy matrix $M$ is independent of $x$. Explicitly,

$$
M=Y^{-1}(x) Y(x+2 \pi) .
$$

A normal solution of (2.1) is any solution $y(x)$ such that $y(x+2 \pi)=\sigma y(x)$, where $\sigma$, called the Floquet multiplier, satisfies the characteristic equation

$$
\sigma^{2}-(\operatorname{tr} M) \sigma+1=0 .
$$

It then follows that (2.1) admits solutions bounded over all $x \in \mathbb{R}$ if and only if

$$
-2 \leqslant \operatorname{tr} M \leqslant 2
$$

(one can easily show that $\operatorname{tr} M \in \mathbb{R}$ ). The values of $\lambda$ for which (2.6) is satisfied comprise the spectrum of the scattering problem. It was shown in [14] that the real $\lambda$ axis divides into bands and gaps, separated by a (finite or infinite) sequence of values $\lambda_{n}$ for $n=1,2 \ldots$ such that $\operatorname{tr} M= \pm 2$.

Our goal is to characterize the properties of the spectrum as a function of $\epsilon$. Since it is not possible to obtain the eigenfunctions (and therefore the monodromy matrix) for (2.1) in closed form, we will use the WKB method [2, 15]. Recall that, in the WKB approach, one looks for an asymptotic expansion for the solution of (2.1) in the form

$$
\phi(x)=A(x) \mathrm{e}^{i S(x) / \epsilon},
$$

with $A(x)$ and $S(x)$ assumed to be $O(1)$ as $\epsilon \rightarrow 0$. Substituting (2.7) into (2.1), one then obtains, at the first two orders in the expansion, the eikonal equation and the transport equation, i.e.,

$$
S^{\prime 2}=Q(x), \quad 2 A^{\prime} S^{\prime}+A S^{\prime \prime}=0,
$$

respectively. These equations are readily integrated to obtain

$$
S(x)=\int_{x_{0}}^{x} \sqrt{Q(y)} \mathrm{d} y, \quad A(x)=\frac{1}{\sqrt[4]{Q(x)}},
$$

up to arbitrary integration constants.

One can now use the above expressions to construct an asymptotic approximation for the fundamental matrix solution of (2.1) that is valid over the whole range $[-\pi, \pi]$. Different values of $\lambda$ lead to different properties for the solutions, however. Explicitly, note that when $\lambda>1, Q(x)$ is positive definite (i.e., $Q(x)>0 \forall x \in \mathbb{R}$ ), whereas 
[Figures were moved to the end of the manuscript in compliance with submission instructions.]

Figure 2. $Q(x)=\lambda-\cos x$ as a function of $x$ over $[-\pi, \pi]$ for $\lambda= \pm 1$ (black curves) and $\lambda=-0.3$ (red curve).

[Figures were moved to the end of the manuscript in compliance with submission instructions.]

Figure 3. The various sub-regions of the fundamental domain $x \in[-\pi, \pi]$ for the WKB analysis in the range $-1<\lambda<1$.

for $\lambda<-1, Q(x)$ is negative definite $(Q(x)<0 \forall x \in \mathbb{R})$. Finally, for $-1<\lambda<1, Q(x)$ is not sign-definite: $Q( \pm c)=0$ with $c=\arccos \lambda$, and one has $Q(x)>0$ for $x \in[-\pi,-c) \cup(c, \pi]$ and $Q(x)<0$ for $x \in(-c, c)$, as illustrated in Fig. 2. It should then be clear, based on the above discussion, that one needs to divide the study of the spectrum into three different ranges: (i) $\lambda<-1$, (ii) $-1<\lambda<1$ and (iii) $\lambda>1$. In each range, we will first obtain asymptotic expressions for the behavior of the eigenfunctions using WKB and then we use these expressions to obtain an asymptotic expression for the monodromy matrix.

\subsection{Range $\lambda<-1$}

For any value of $\lambda$ in this range, $Q(x)$ is negative definite. Two linearly independent solutions are given, in the WKB approximation, by

$$
y_{1}(x)=\frac{1}{\sqrt[4]{-Q(x)}} \exp \left[\int_{-\pi}^{x} \sqrt{-Q(s)} \mathrm{d} s / \epsilon\right], \quad y_{2}(x)=\frac{1}{\sqrt[4]{-Q(x)}} \exp \left[-\int_{-\pi}^{x} \sqrt{-Q(s)} \mathrm{d} s / \epsilon\right],
$$

and we can write a fundamental matrix solution $Y(x)$ as (2.3) with $y_{1}(x)$ and $y_{2}(x)$ as in (2.10). Such a solution is uniformly valid over the whole range $x \in[-\pi, \pi]$. The value of this fundamental matrix solution at $x=-\pi$ and at $x=\pi$ is, respectively,

$$
Y(-\pi)=\left(\begin{array}{cc}
\frac{1}{\sqrt[4]{-\lambda-1}} & \frac{1}{\sqrt[4]{-\lambda-1}} \\
\frac{\sqrt[4]{-\lambda-1}}{\epsilon} & -\frac{\sqrt[4]{-\lambda-1}}{\epsilon}
\end{array}\right), \quad Y(\pi)=\left(\begin{array}{cc}
\frac{1}{\sqrt[4]{-\lambda-1}} \mathrm{e}^{S_{\mathrm{i}} / \epsilon} & \frac{1}{\sqrt[4]{-\lambda-1}} \mathrm{e}^{-S_{\mathrm{i}} / \epsilon} \\
\frac{\sqrt[4]{-\lambda-1}}{\epsilon} \mathrm{e}^{S_{\mathrm{i}} / \epsilon} & -\frac{\sqrt[4]{-\lambda-1}}{\epsilon} \mathrm{e}^{-S_{\mathrm{i}} / \epsilon}
\end{array}\right)
$$

where

$$
S_{\mathrm{i}}(\lambda)=\int_{-\pi}^{\pi} \sqrt{-Q(x)} \mathrm{d} x=4 \sqrt{1-\lambda} E\left(\sqrt{\frac{2}{1-\lambda}}\right) .
$$

The mondoromy matrix can be obtained as $M=Y^{-1}(-\pi) Y(\pi)$, and its trace is given by

$$
\operatorname{tr} M=2 \cosh \left(S_{\mathrm{i}} / \epsilon\right) .
$$

Note that $\operatorname{tr} M>2$ for all $\lambda<-1$. Therefore, for any value of $\lambda$ in this range the scattering problem does not admit bounded solutions. Thus, all values $\lambda<-1$ do not belong to the spectrum of the scattering problem.

\subsection{Range $\lambda>1$}

For any value of $\lambda$ in this range, $Q(x)$ is positive definite. Consequently, two linearly independent solutions are, in the WKB approximation,

$$
y_{1}(x)=\frac{1}{\sqrt[4]{Q(x)}} \exp \left[i \int_{-\pi}^{x} \sqrt{Q(s)} \mathrm{d} s / \epsilon\right], \quad y_{2}(x)=\frac{1}{\sqrt[4]{Q(x)}} \exp \left[-i \int_{-\pi}^{x} \sqrt{Q(s)} \mathrm{d} s / \epsilon\right] .
$$

The corresponding fundamental matrix solution is still given by (2.3), but with $y_{1}(x)$ and $y_{2}(x)$ now given by (2.14). The value of the fundamental matrix solution at $x=-\pi$ and $x=\pi$ is, respectively,

$$
Y(-\pi)=\left(\begin{array}{cc}
\frac{1}{\sqrt[4]{\lambda+1}} & \frac{1}{\sqrt[4]{\lambda+1}} \\
\frac{\sqrt[4]{\lambda+1}}{\epsilon} & -\frac{\sqrt[4]{\lambda+1}}{\epsilon}
\end{array}\right), \quad Y(\pi)=\left(\begin{array}{cc}
\frac{1}{\sqrt[4]{\lambda+1}} \mathrm{e}^{i S_{\mathrm{iii}} / \epsilon} & \frac{1}{\sqrt[4]{\lambda+1}} \mathrm{e}^{-i S_{\mathrm{iii}} / \epsilon} \\
\frac{\sqrt[4]{\lambda+1}}{\epsilon} \mathrm{e}^{i S_{\mathrm{iii}} / \epsilon} & -\frac{\sqrt[4]{\lambda+1}}{\epsilon} \mathrm{e}^{-i S_{\mathrm{iii}} / \epsilon}
\end{array}\right)
$$


where

$$
S_{\mathrm{iii}}(\lambda)=\int_{-\pi}^{\pi} \sqrt{Q(x)} \mathrm{d} x=4 \sqrt{1+\lambda} E\left(\sqrt{\frac{2}{1+\lambda}}\right) .
$$

The mondoromy matrix is given by $M=Y^{-1}(-\pi) Y(\pi)$ as before, but its trace is now

$$
\operatorname{tr} M=2 \cos \left(S_{\mathrm{iii}} / \epsilon\right) .
$$

Note that $|\operatorname{tr} M| \leqslant 2$. Therefore all values of $\lambda$ in this range belong to the spectrum. In other words, the range $\lambda \in(1, \infty)$ comprises one infinitely long stable band.

\subsection{Range $-1<\lambda<1$}

This is the most interesting portion of the spectrum, and as a result also the most complicated range. Recall that, for any $\lambda$ in this range, we have $Q( \pm c)=0$, where $c=\arccos \lambda$. Also recall that the zeroes of $Q(x)$ are known as the turning points. They are the boundaries of the forbidden regions for the motion in classical mechanics. In the quantum-mechanical analogy, the behavior of the eigenfunctions changes from oscillating to exponential (or viceversa) as one crosses a turning point. Correspondingly, the validity of the WKB approximation breaks down in the vicinity of a turning point. Therefore one needs to study the neighborhood of the turning points individually.

We will first discuss the behavior of the eigenfunctions in the following sub-regions of the fundamental period $x \in[-\pi, \pi]$ : (a) Region 1, $x \in[-\pi,-c)$; (b) First transition region, comprised by a neighborhood of $x=-c$; (c) Region 2, $x \in(-c, c)$, (d) Second transition region, comprised by a neighborhood of $x=c$; (e) Region $3, x \in$ $(c, \pi]$. The various sub-regions are illustrated in Fig. 3. We will then need to relate the behavior of the eigenfunctions across adjacent sub-regions using matched asymptotic expansions.

\subsubsection{WKB solutions in the individual sub-regions}

The calculation of the WKB approximations for the general solution of the scattering problem in each of the individual sub-regions 1, 2 and 3 is essentially similar to those in section 2.1 and 2.2. The calculation of an asymptotic approximation for the general solution in the neighborhood of each transition point is done via standard methods (e.g., see [2]). Omitting the details for brevity, the results are as follows:

(a) In region $1, x \in[-\pi,-c)$, the general solution of the scattering problem in the WKB approximation is

$$
y_{g, 1}(x)=a_{1}^{-} y_{1}(x)+a_{1}^{+} y_{2}(x),
$$

where

$$
\begin{gathered}
y_{1}(x)=\frac{1}{\sqrt[4]{Q(x)}} \mathrm{e}^{i S_{1} / \epsilon}, \quad y_{2}(x)=\frac{1}{\sqrt[4]{Q(x)}} \mathrm{e}^{-i S_{1} / \epsilon}, \\
S_{1}(x, \lambda)=-\int_{-c}^{x} \sqrt{Q(s)} \mathrm{d} s .
\end{gathered}
$$

Later on, we will need a distinguished value of $S_{1}(x, \lambda)$ :

$$
S_{1}(\lambda)=S_{1}(-\pi, \lambda)=2 \sqrt{1+\lambda} E\left(\frac{\pi}{2}-\frac{1}{2} \arccos (\lambda), \sqrt{\frac{2}{1+\lambda}}\right),
$$

where $E(\phi, k)$ is the incomplete elliptic integral of the second kind [18]. A plot of $S_{1}(-\pi, \lambda)$ as a function of $\lambda$ is given in the first of Fig. 4.

(b) In the first transition region, $x \in(-c-\delta,-c+\delta)$ with $\delta>0$, and an asymptotic expression for the solution is

$$
y_{g, 1 \rightarrow 2}(x)=c_{1}^{-} \mathrm{Ai}[\xi(x)]+c_{1}^{+} \operatorname{Bi}[\xi(x)],
$$

where

$$
\xi(x)=a^{1 / 3}(x+c) / \epsilon^{2 / 3}, \quad a=\sqrt{1-\lambda^{2}} .
$$


(c) The WKB approximation to the general solution in region $2, x \in(-c, c)$, is given equivalently by either

or

$$
y_{g, 2}(x)=\frac{1}{\sqrt[4]{|Q(x)|}}\left(a_{2}^{-} \mathrm{e}^{-S_{2} / \epsilon}+a_{2}^{+} \mathrm{e}^{S_{2} / \epsilon}\right),
$$

$$
y_{g, 2}(x)=\frac{1}{\sqrt[4]{|Q(x)|}}\left(\bar{a}_{2}^{-} \mathrm{e}^{-\bar{S}_{2} / \epsilon}+\bar{a}_{2}^{+} \mathrm{e}^{\bar{S}_{2} / \epsilon}\right),
$$

with

$$
S_{2}(x, \lambda)=\int_{-c}^{x} \sqrt{-Q(s)} \mathrm{d} s, \quad \bar{S}_{2}(x, \lambda)=\int_{c}^{x} \sqrt{-Q(s)} \mathrm{d} s .
$$

Again, later on we will need a distinguished value of $S_{2}(x, \lambda)$ :

$$
S_{2}(\lambda)=S_{2}(c, \lambda)=4 \sqrt{1-\lambda} E\left(\frac{1}{2} \arccos (\lambda), \sqrt{\frac{2}{1-\lambda}}\right) .
$$

A plot of $S_{2}(c, \lambda)$ as a function of $\lambda$ is given in the right panel of Fig. 4 .

(d) An asymptotic expansion for the solution in the second transition region, $x \in(c-\delta, c+\delta)$, is given by

$$
y_{g, 2 \rightarrow 3}(x)=c_{2}^{-} \mathrm{A} i(z)+c_{2}^{+} \mathrm{Bi}(z), \quad z=-a^{1 / 3}(x-c) / \epsilon^{2 / 3} .
$$

(e) The WKB approximation to the general solution in region $3, x \in(c, \pi]$, is given by

$$
y_{g, 3}=\frac{1}{\sqrt[4]{Q(x)}}\left(a_{3}^{-} \mathrm{e}^{-i S_{3} / \epsilon}+a_{3}^{+} \mathrm{e}^{i S_{3} / \epsilon}\right), \quad S_{3}(x, \lambda)=\int_{c}^{x} \sqrt{Q(s)} \mathrm{d} s .
$$

\subsubsection{Matching the solutions across sub-regions: connection formulae}

All the above expressions for the general solution in each of the sub-regions contains two arbitrary constants. To obtain a representation for the solution of the scattering problem that remains valid over all of the fundamental domain $x \in[-\pi, \pi]$, one must be able to continue a given solution in each sub-region to that in the next one. This is done by relating the values of the constant in one sub-region to those in the next one, resulting in the so-called transition (or connection) formulae (e.g., see [2]).

First we match $y_{g, 1}(x)$ with $y_{g, 1 \rightarrow 2}(x)$. Importantly, the asymptotic expression for the solution in the transition region is valid for $|x+c|=o(1)$, while that for the solution in region 1 becomes ill-ordered only when $|x+c|=$ $o\left(\epsilon^{2 / 3}\right)[2]$. Thus, the region of validity of the solution in the first transition region overlaps with the region of validity of the solution in region 1 . To match the solutions, one then computes the asymptotic behavior of both solutions in the overlapping region, by taking $|x+c| \rightarrow 0$ and $\xi \rightarrow-\infty$. Explicitly,

$$
\begin{gathered}
y_{g, 1}(x)=\frac{1}{\sqrt[4]{a|x+c|}}\left(a_{1}^{-} \mathrm{e}^{\frac{2}{3} i \sqrt{a}|x+c|^{3 / 2} / \epsilon}+a_{1}^{+} \mathrm{e}^{-\frac{2}{3} i \sqrt{a}|x+c|^{3 / 2} / \epsilon}\right)(1+O(x+c)), \quad x \rightarrow-c, \\
y_{g, 1 \rightarrow 2}(x)=\frac{1}{\sqrt[4]{\pi^{2}|\xi|}}\left[c_{1}^{-} \sin \left(\frac{2}{3}|\xi|^{3 / 2}+\frac{\pi}{4}\right)+c_{1}^{+} \cos \left(\frac{2}{3}|\xi|^{3 / 2}+\frac{\pi}{4}\right)\right]\left(1+O\left(1 /|\xi|^{3 / 2}\right)\right), \quad \xi \rightarrow-\infty .
\end{gathered}
$$

Requiring that these two expansions match, we obtain the connection formula

$$
\left(\begin{array}{c}
c_{1}^{+} \\
c_{1}^{-}
\end{array}\right)=C_{1}\left(\begin{array}{l}
a_{1}^{+} \\
a_{1}^{-}
\end{array}\right), \quad C_{1}=\frac{\sqrt{\pi}}{(a \epsilon)^{1 / 6}}\left(\begin{array}{cc}
\mathrm{e}^{i \pi / 4} & \mathrm{e}^{-i \pi / 4} \\
-i \mathrm{e}^{i \pi / 4} & i \mathrm{e}^{-i \pi / 4}
\end{array}\right) .
$$

[Figures were moved to the end of the manuscript in compliance with submission instructions.]

Figure 4. Left: $S_{1}(\lambda)$ as a function of $\lambda$ (red curve). Right: $S_{2}(\lambda)$ as a function of $\lambda$ (red curve). The solid gray curves show the linear approximation of $S_{1}(\lambda)$ and $S_{2}(\lambda)$ about $\lambda=-1$ and $\lambda=1$, respectively. The black curve shows the second-order Taylor expansion of $S_{1}(\lambda)$ about $\lambda=-1$. The dashed gray horizontal lines show the levels $S_{1}(\lambda)=\frac{1}{2}\left(n-\frac{1}{2}\right) \pi \epsilon$, where $n=1,2 \ldots$, for $\epsilon=0.169$ (corresponding to the ZK experiment; cf. section 5 for details). The dashed vertical line shows the threshold value $S_{2}(\lambda)=\epsilon \ln \left(\frac{2}{\pi \kappa}\right)$, where $\kappa=0.01$. 
In other words, the matrix $C_{1}$ allows one to obtain the coefficients $c_{1}^{ \pm}$of the particular solution in the first transition region that is the continuation of the solution in region 1 with a given choice of coefficients $a_{1}^{ \pm}$.

The matching of the solutions across the other regions is accomplished using the same approach. To match $y_{g, 2}(x)$ with $y_{g, 1 \rightarrow 2}(x)$, note that

$$
\begin{gathered}
y_{g, 2}(x)=\frac{1}{\sqrt[4]{a|x+c|}}\left(a_{2}^{-} \mathrm{e}^{-\frac{2}{3} \sqrt{a}(x+c)^{3 / 2} / \epsilon}+a_{2}^{+} \mathrm{e}^{\frac{2}{3} \sqrt{a}(x+c)^{3 / 2} / \epsilon}\right)(1+O(x+c)), \quad x \rightarrow-c, \\
y_{g, 1 \rightarrow 2}(\xi)=\frac{1}{\sqrt[4]{\pi^{2} \xi}}\left(\frac{1}{2} c_{1}^{-} \mathrm{e}^{-\frac{2}{3} \xi^{3 / 2}}+c_{1}^{+} \mathrm{e}^{\frac{2}{3} \xi^{3 / 2}}\right)\left(1+O\left(1 / \xi^{3 / 2}\right)\right), \quad \xi \rightarrow \infty .
\end{gathered}
$$

Matching these expansions, we obtain

$$
\left(\begin{array}{l}
a_{2}^{+} \\
a_{2}^{-}
\end{array}\right)=C_{2}\left(\begin{array}{l}
c_{1}^{+} \\
c_{1}^{-}
\end{array}\right), \quad C_{2}=\frac{(a \epsilon)^{1 / 6}}{\sqrt{\pi}}\left(\begin{array}{ll}
1 & 0 \\
0 & \frac{1}{2}
\end{array}\right) .
$$

Note also that $\bar{a}_{2}^{ \pm}$in (2.25) relates to $a_{2}^{ \pm}$in (2.24) by

$$
\left(\begin{array}{l}
\bar{a}_{2}^{+} \\
\bar{a}_{2}^{-}
\end{array}\right)=C_{3}\left(\begin{array}{l}
a_{2}^{+} \\
a_{2}^{-}
\end{array}\right), \quad C_{3}=\left(\begin{array}{cc}
\mathrm{e}^{S_{2}(c, \lambda) / \epsilon} & 0 \\
0 & \mathrm{e}^{-S_{2}(c, \lambda) / \epsilon}
\end{array}\right) .
$$

To match $y_{g, 2 \rightarrow 3}(x)$ with $y_{g, 2}(x)$, note that

$$
\begin{gathered}
y_{g, 2}(x)=\frac{1}{\sqrt[4]{a|x-c|}}\left(\bar{a}_{2}^{-} \mathrm{e}^{\frac{2}{3} \sqrt{a}|x-c|^{3 / 2} / \epsilon}+\bar{a}_{2}^{+} \mathrm{e}^{-\frac{2}{3} \sqrt{a}|x-c|^{3 / 2} / \epsilon}\right)(1+O(|x-c|)), \quad x \rightarrow c, \\
y_{g, 2 \rightarrow 3}(z)=\frac{1}{\sqrt[4]{\pi^{2} z}}\left(\frac{1}{2} c_{2}^{-} \mathrm{e}^{-\frac{2}{3} z^{3 / 2}}+c_{2}^{+} \mathrm{e}^{\frac{2}{3} z^{3 / 2}}\right)\left(1+O\left(1 / z^{3 / 2}\right)\right), \quad z \rightarrow \infty .
\end{gathered}
$$

Matching these expansions, we obtain

$$
\left(\begin{array}{l}
c_{2}^{-} \\
c_{2}^{+}
\end{array}\right)=C_{4}\left(\begin{array}{l}
\bar{a}_{2}^{+} \\
\bar{a}_{2}^{-}
\end{array}\right), \quad C_{4}=\frac{\sqrt{\pi}}{(a \epsilon)^{1 / 6}}\left(\begin{array}{ll}
2 & 0 \\
0 & 1
\end{array}\right) .
$$

Finally, to match $y_{g, 3}(x)$ with $y_{g, 2 \rightarrow 3}(x)$, note that

$$
\begin{gathered}
y_{g, 3}=\frac{1}{\sqrt[4]{a(x-c)}}\left(a_{3}^{-} \mathrm{e}^{-i \frac{2}{3} \sqrt{a}(x-c)^{3 / 2} / \epsilon}+a_{3}^{+} \mathrm{e}^{i \frac{2}{3} \sqrt{a}(x-c)^{3 / 2} / \epsilon}\right)(1+O(x-c)), \quad x \rightarrow c, \\
y_{g, 2 \rightarrow 3}=\frac{1}{\sqrt[4]{\pi^{2}|z|}}\left[c_{2}^{-} \sin \left(\frac{2}{3}|z|^{3 / 2}+\frac{\pi}{4}\right)+c_{2}^{+} \cos \left(\frac{2}{3}|z|^{3 / 2}+\frac{\pi}{4}\right)\right]\left(1+O\left(1 /|z|^{3 / 2}\right)\right), \quad z \rightarrow-\infty .
\end{gathered}
$$

Requiring that these expansions match, we obtain

$$
\left(\begin{array}{l}
a_{3}^{+} \\
a_{3}^{-}
\end{array}\right)=C_{5}\left(\begin{array}{l}
c_{2}^{-} \\
c_{2}^{+}
\end{array}\right), \quad C_{5}=\frac{(a \epsilon)^{1 / 6}}{2 \sqrt{\pi}}\left(\begin{array}{cc}
-i \mathrm{e}^{i \pi / 4} & \mathrm{e}^{i \pi / 4} \\
i \mathrm{e}^{-i \pi / 4} & \mathrm{e}^{-i \pi / 4}
\end{array}\right) .
$$

We are now ready to combine all of the above results. The overall connection matrix $C$, which relates the coefficients of the solution for $x \in[-\pi,-c)$ to those of the solution for $x \in(c, \pi]$, is given by

where

$$
C=C_{5} C_{4} C_{3} C_{2} C_{1}=\cosh \left[S_{2, \epsilon}(\lambda) / \epsilon\right] I+\sinh \left[S_{2, \epsilon}(\lambda) / \epsilon\right] \sigma_{2},
$$

$$
S_{2, \epsilon}(\lambda)=S_{2}(\lambda)+\epsilon \log 2, \quad \sigma_{2}=\left(\begin{array}{cc}
0 & -i \\
i & 0
\end{array}\right) .
$$

That is, the matrix $C$ allows one to compute the coefficients $a_{3}^{ \pm}$of the solution in region 3 that is the continuation of the solution in region 1 with a given choice of coefficients $a_{1}^{ \pm}$. 


\subsubsection{Trace of the monodromy matrix}

We now use the above results to compute the monodromy matrix. In the sub-region $x \in[-\pi,-c)$ we choose the fundamental matrix solution $Y(x)$ as in (2.3) with $y_{1}(x)$ and $y_{2}(x)$ given by (2.19). The value of the above the fundamental matrix solution at $x=-\pi$ is thus

$$
Y(-\pi)=\left(\begin{array}{cc}
\frac{1}{\sqrt[4]{1+\lambda}} \mathrm{e}^{-i S_{1}(\lambda) / \epsilon} & \frac{1}{\sqrt[4]{1+\lambda}} \mathrm{e}^{i S_{1}(\lambda) / \epsilon} \\
\frac{i \sqrt[4]{1+\lambda}}{\epsilon} \mathrm{e}^{-i S_{1}(\lambda) / \epsilon} & \frac{-i \sqrt[4]{1+\lambda}}{\epsilon} \mathrm{e}^{i S_{1}(\lambda) / \epsilon}
\end{array}\right)
$$

Based on the above discussion, the value of the continuation of the above fundamental matrix solution at $x=\pi$ solution is given by

$$
Y(\pi)=\left(\begin{array}{cc}
\frac{1}{\sqrt[4]{1+\lambda}} \mathrm{e}^{i S_{1}(\lambda) / \epsilon} & \frac{1}{\sqrt[4]{1+\lambda}} \mathrm{e}^{-i S_{1}(\lambda) / \epsilon} \\
\frac{i \sqrt[4]{1+\lambda}}{\epsilon} \mathrm{e}^{i S_{1}(\lambda) / \epsilon} & \frac{-i \sqrt[4]{1+\lambda}}{\epsilon} \mathrm{e}^{-i S_{1}(\lambda) / \epsilon}
\end{array}\right) C,
$$

where $C$ is the connnection matrix in (2.43). Recall now that we can obtain the monodromy matrix simply as $M=$ $Y^{-1}(-\pi) Y(\pi)$. Straightforward algebra then shows that the trace of the monodromy matrix is given by

$$
\operatorname{tr} M=2 \cosh \left[S_{2, \epsilon}(\lambda) / \epsilon\right] \cos \left(2 S_{1}(\lambda) / \epsilon\right),
$$

with $S_{1}(\lambda)$ and $S_{2, \epsilon}(\lambda)$ given by (2.21) and (2.44), respectively.

\section{Asymptotic expressions for band widths, gap widths and number of bands}

We now use the results of the previous sections to characterize the spectrum of the scattering problem for the KdV equation with cosine initial condition as $\epsilon \rightarrow 0$. As an example, Fig. 5 shows a plot of $\operatorname{tr} M$ with $\epsilon=0.169$. Note that, as in [19], in order to capture the whole behavior of the trace function in a single plot it is convenient to plot the function $f(\operatorname{tr} M / 2)$ instead of $\operatorname{tr} M$ itself, where

$$
f(\Delta)= \begin{cases}\Delta, & |\Delta| \leq 1, \\ \operatorname{sgn} \Delta(1+\log |\Delta|), & |\Delta|>1\end{cases}
$$

Recall that the spectrum of the scattering problem is given by all values of $\lambda$ for which $-2 \leqslant \operatorname{tr} M \leqslant 2$, and that for $-1<\lambda<1$ the trace function $\operatorname{tr} M$ is given by (2.47). The $1 / \epsilon$ in the argument of the cosine implies that, as $\epsilon \rightarrow 0, \operatorname{tr} M$ becomes a rapidly oscillating function of $\lambda$. At the same, the $1 / \epsilon$ in the argument of the hyperbolic cosine implies that, as $\epsilon \rightarrow 0$, the amplitude of these oscillations grows exponentially. Therefore as $\epsilon \rightarrow 0$, the values of $\lambda$ for which $\operatorname{tr} M= \pm 2$, which comprise the edges of the bands and gaps of the spectrum, will be closely clustered near the zeros $z_{n}$ of $\operatorname{tr} M$. It is trivial to see that these zeros are given by the solutions of

$$
S_{1}\left(z_{n}\right)=\frac{1}{2}\left(n-\frac{1}{2}\right) \pi \epsilon,
$$

for $n=1,2,3 \ldots$ Since $S_{1}(\lambda)$ is a monotonically increasing function and $\max \left[S_{1}(\lambda)\right]=S_{1}(1)=2 \sqrt{2}$, the total number of zeros is given by

$$
N_{\text {zeros }}=\left\lfloor\frac{4 \sqrt{2}}{\pi \epsilon}+\frac{1}{2}\right\rfloor .
$$

We can also obtain an analytical expression for the approximate location of the zeros, by considering the Taylor expansion of $S_{1}(\lambda)$ about $\lambda=-1$

$$
S_{1}(\lambda)=\frac{\pi}{2 \sqrt{2}}(\lambda+1)+\frac{\pi}{32}(\lambda+1)^{2}+O(\lambda+1)^{3}
$$

Substituting (3.4) into (3.2) and keeping linear or quadratic terms, we then obtain a linear or a quadratic approximation of the zeros of the trace function,

$$
z_{n, \text { linear }}=-1+\sqrt{2}\left(n-\frac{1}{2}\right) \epsilon, \quad z_{n, \text { quadratic }}=-1-4 \sqrt{2}+\sqrt{8(2 n-1) \epsilon+32} .
$$


[Figures were moved to the end of the manuscript in compliance with submission instructions.]

Figure 5. The function $f(\operatorname{tr} M / 2)$ defined by (3.1) as a function of $\lambda \in[-1,1]$ for $\epsilon=0.169$ (corresponding to the ZK experiment; cf. section 5 for details). Dash-dotted red curve: the expression (2.47) obtained via WKB. Solid blue curve: results from numerical integration of the scattering problem (cf. section 5 for details). (Note that the WKB results are almost indistinguishable from those from full numerics, and as a result the blue curve is hidden by the red curve.) Black curves (dashed): the envelope function $\pm \cosh \left[S_{2, \epsilon}(\lambda) / \epsilon\right]$ from (2.47). The dotted vertical line denotes the solitonic threshold $\lambda_{s}$ (cf. section 3 for details).

Recall that the real $\lambda$ axis divides into bands and gaps, separated by a sequence of values $\lambda_{n}$ such that $\operatorname{tr} M= \pm 2$. The zeros $z_{n}$ characterize the points where the main spectrum clusters in the limit of small $\epsilon$, and hence give an asymptotic estimate of the total number of nonlinear excitations of the problem. Note, however, that not all of these excitations resemble solitons of the infinite line problem. In order to distinguish the soliton-like excitations from the non-solitonic ones, one must go one step further and characterize how closely each spectral band is clustered around the corresponding zero. In other words, one must compute the relative width of each band and gap.

The width of the $n$-th spectral band, which is approximately centered at $z_{n}$, is given by $w_{n}=\lambda_{2 n}-\lambda_{2 n-1}$, whereas the width of the $n$-th spectral gap is given by $g_{n}=\lambda_{2 n+1}-\lambda_{2 n}$. The more relevant quantity for our purposes, however, is the relative band width $W_{n}$, defined as the ratio between the band width and the sum of the band width and gap width, or equivalently the relative gap width $G_{n}$, which is the complement to 1 of the relative band width. That is, $W_{n}$ and $G_{n}$ are given by

$$
W_{n}=\frac{w_{n}}{w_{n}+g_{n}}=\frac{\lambda_{2 n}-\lambda_{2 n-1}}{\lambda_{2 n+1}-\lambda_{2 n-1}}, \quad G_{n}=1-W_{n}=\frac{\lambda_{2 n+1}-\lambda_{2 n}}{\lambda_{2 n+1}-\lambda_{2 n-1}} .
$$

To obtain an asymptotic characterization of the spectrum, we first consider the Taylor expansion of $\operatorname{tr} M$ around $z_{n}$, evaluated at $\lambda=\lambda_{2 n}$ and $\lambda=\lambda_{2 n-1}$. Because of the exponentially large oscillations of the trace function, it is sufficient to only keep linear terms. By doing so we can approximate the condition $\pm \operatorname{tr} M= \pm 2$ as $2=\left|(\operatorname{tr} M)^{\prime}\left(z_{n}\right)\right|\left(z_{n}-\lambda_{2 n-1}\right)+O\left(z_{n}-\lambda_{2 n-1}\right)^{2}=\left|(\operatorname{tr} M)^{\prime}\left(z_{n}\right)\right|\left(\lambda_{2 n}-z_{n}\right)+O\left(\lambda_{2 n}-z_{n}\right)^{2}$. Combining these expressions and recalling (2.47), we can then write the $n$-th band width as

$$
w_{n}=\frac{\epsilon}{\left|S_{1}^{\prime}\left(z_{n}\right)\right|} \operatorname{sech}\left[S_{2, \epsilon}\left(z_{n}\right) / \epsilon\right]+O\left(\epsilon \mathrm{e}^{-2 S_{2}\left(z_{n}\right) / \epsilon}\right)
$$

Next we use the difference $z_{n+1}-z_{n}$, which is the distance between two adjacent zeros of tr $M$, to approximate $w_{n}+g_{n}$. It is straightforward to show that the difference between these two quantities is given by

$$
\left(w_{n}+g_{n}\right)-\left(z_{n+1}-z_{n}\right)=\frac{1}{2}\left(w_{n}-w_{n+1}\right)+O\left(\epsilon \mathrm{e}^{-2 S_{2}\left(z_{n+1}\right) / \epsilon}\right)=O\left(\epsilon \mathrm{e}^{-S_{2}\left(z_{n+1}\right) / \epsilon}\right) .
$$

Also note that $S_{1}\left(\lambda_{n+1}\right)-S_{1}\left(\lambda_{n}\right)=\frac{1}{2} \pi \epsilon$. We then expand $S_{1}(\lambda)$ around $z_{n}$, evaluate at $\lambda=z_{n+1}$ and obtain

$$
z_{n+1}-z_{n}=\frac{\pi \epsilon}{2\left|S_{1}^{\prime}\left(z_{n}\right)\right|}+O\left(\epsilon^{2}\right)
$$

Combining these results, we then obtain

$$
w_{n}+g_{n}=\frac{\pi \epsilon}{2\left|S_{1}^{\prime}\left(z_{n}\right)\right|}+O\left(\epsilon^{2}\right)
$$

Finally, combining (3.7) and (3.10), we obtain an asymptotic expression for the $n$-th relative band width as

$$
W_{n}=\frac{2}{\pi} \operatorname{sech}\left[S_{2, \epsilon}\left(z_{n}\right) / \epsilon\right]+O\left(\epsilon \mathrm{e}^{-S_{2}\left(z_{n}\right) / \epsilon}\right) .
$$




\section{Asymptotic characterization of the spectrum}

We now use the results of Section 3 to characterize analytically the asymptotic properties of the spectrum, including the number and the amplitude of the solitons. Recall that, in the inverse scattering transform for the KdV equation with periodic boundary conditions, the reconstruction formula is given by

$$
u(x, t)=\sum_{n=1}^{2 N+1} \lambda_{n}-2 \sum_{n=1}^{N} \mu_{n}
$$

where the $\lambda_{n}$ comprise the main spectrum and $\mu_{n}$ comprise the auxiliary spectrum, and $N$ is the number of unstable bands. In other words, the $\lambda_{n}$ are the endpoints of the stable bands; $\lambda_{n}$ is the $n$-th band edge and $\mu_{n} \in\left[\lambda_{2 n-1}, \lambda_{2 n}\right]$ satisfies the Dubrovin equations. In the case of a genus-1 (i.e., elliptic) solution, (4.1) reduces to the cnoidal wave of the $\mathrm{KdV}$ equation [1]:

$$
u(x, t)=\lambda_{1}+\lambda_{3}-\lambda_{2}-2\left(\lambda_{3}-\lambda_{2}\right) \mathrm{cn}^{2}\left(\sqrt{\lambda_{3}-\lambda_{1}}\left(x-2\left(\lambda_{1}+\lambda_{2}+\lambda_{3}\right) t-x_{0}\right) \mid m\right),
$$

where $\operatorname{cn}(\phi \mid m)$ is the Jacobian elliptic cosine with amplitude $\phi$ and elliptic modulus $m$, which is given by $m=$ $\left(\lambda_{3}-\lambda_{2}\right) /\left(\lambda_{3}-\lambda_{1}\right)$.

The solitary wave limit of (4.2) is then obtained by considering the limit $\lambda_{1} \rightarrow \lambda_{2}$. More in general, the solitonic limit of (4.1) is obtained by taking the limit $\lambda_{2 n-1} \rightarrow \lambda_{2 n}$ for all $n=1, \ldots, N$. In the periodic problem, however, one will always have $\lambda_{2 n-1}<\lambda_{2 n}$ for all $n=1, \ldots, N$ and for all $\epsilon>0$, since $\lambda_{2 n-1}$ and $\lambda_{2 n}$ are defined by the conditions $\operatorname{tr}\left[M\left(\lambda_{2 n-1}\right)\right]=2$ and $\operatorname{tr}\left[M\left(\lambda_{2 n}\right)\right]=-2$, and $\operatorname{tr} M$ is an entire function of $\lambda$ [14]. Therefore, one never has true solitons in the periodic problem. Nonetheless, excitations with modulus sufficiently close to 1 are good approximations of the solitons of the $\mathrm{KdV}$ equation, and the match becomes closer and closer as the modulus tends to 1 .

Accordingly, chosen a fixed threshold $\kappa \ll 1$, we define a nonlinear excitation of the periodic problem to be an effective soliton if its relative band width is less than $\kappa$. Note that $\kappa$ is just the complement to 1 of the so-called "soliton index" $\bar{\kappa}[3]$ or equivalently of the "running index" [19]; i.e., $\mathcal{\kappa}+\bar{\kappa}=1$. In the next section we will exploit the results of the previous sections to characterize the number and amplitude of effective solitons as $\epsilon \rightarrow 0$. It is important to emphasize that, while the introduction of the artificial and arbitrary parameter $\kappa$ might seem unsatisfactory, in the end we will see that the precise value of $\kappa$ is immaterial in the limit $\epsilon \rightarrow 0$.

\subsection{Number of solitons}

Recall that (3.11) provides an asymptotic expression for the relative band width $W_{n}$ (or equivalently the relative gap width $G_{n}=1-W_{n}$ ). Solving the inequality $W_{n}<\kappa$ (or equivalently $G_{n}>\bar{\kappa}$ ) we then see that, as $\epsilon \rightarrow 0$, the solitonic excitations are confined to the range $\lambda \in\left(-1, \lambda_{s}\right)$, where $\lambda_{s}$ is implicitly defined by the equation

$$
S_{2}\left(\lambda_{s}\right)=\epsilon \ln \frac{2}{\pi \kappa}
$$

obtained from the leading order by expansion of (3.11). Since $S_{2}(\lambda)$ is a monotonically decreasing function (cf. Fig. 4), there exists an unique $\lambda_{s}$ satisfying (4.3). While there is no simple closed-form expression for $S_{2}(\lambda)$ or its inverse, one can easily find the value of $\lambda_{s}$ numerically. One can also find an analytic approximation with a linear expansion of $S_{2}(\lambda)$ near $\lambda=1$

$$
S_{2}(\lambda)=-\frac{\pi}{\sqrt{2}}(\lambda-1)+o(\lambda-1)^{2}
$$

Substituting (4.4) into (4.3), we then obtain the approximate value of $\lambda_{s}$

$$
\lambda_{s, \text { approx }}=1-\epsilon \frac{\sqrt{2}}{\pi} \ln \left(\frac{2}{\pi \kappa}\right) .
$$

Note that the difference between $\lambda_{s}$ and $\lambda_{s, \text { approx }}$ goes to zero in the limit $\epsilon \rightarrow 0$. 
Correspondingly, the number of solitons equals the number of the zeros of the trace function in the interval $\left(-1, \lambda_{s}\right)$. Recalling the discussion in the previous section, one finds that such a number is given by

$$
N_{\text {soliton }}=\left\lfloor\frac{2 S_{1}\left(\lambda_{s}\right)}{\pi \epsilon}+\frac{1}{2}\right\rfloor
$$

Substituting the Taylor expansion (3.4) of $S_{1}(\lambda)$ into (4.6) and keeping linear or quadratic terms, and using (4.5), we can also obtain a linear or a quadratic approximation for the number of the solitons as a function of $\epsilon$ as

$$
\begin{gathered}
N_{s, \text { linear }}=\left\lfloor\frac{\sqrt{2}}{\epsilon}-\frac{1}{\pi} \ln \left(\frac{2}{\pi \kappa}\right)+\frac{1}{2}\right\rfloor, \\
N_{s, \text { quadratic }}=\left\lfloor\left(\sqrt{2}+\frac{1}{4}\right) \frac{1}{\epsilon}-\left(1+\frac{\sqrt{2}}{4}\right) \frac{1}{\pi} \ln \left(\frac{2}{\pi \kappa}\right)+\frac{1}{2}\right\rfloor .
\end{gathered}
$$

From (4.7) we see immediately that both $N_{s, \text { linear }}$ and $N_{s, \text { quadratic }}$ are independent of the threshold $\kappa$ to leading order. Hence the particular value chosen for $\kappa$ becomes progressively less relevant as $\epsilon \rightarrow 0$. Nonetheless, in section 5 we will show that (4.7b) yields a more accurate estimate for the number of solitons (cf. Fig. 7).

Recall that Zabusky's empirical estimate for the number of solitons is $N_{S} \sim 0.2 / \delta[30,31]$ (where $\delta^{2}$ is the dispersion coefficient in Zabusky and Kruskal's normalizations for the KdV equation), which is equivalent to $N_{s} \sim$ $1.54 / \epsilon$ (see section 5 for details). Comparing Zabusky's estimate with either of the two expressions (4.7) we see that Zabusky's estimates is only an approximation to the leading order term. More precisely, the relative difference between Zabusky's estimate and the leading order term of either of (4.7) is $8 \%$.

\subsection{Soliton amplitudes}

One can easily see from (4.2) that the amplitude of a genus-1 nonlinear excitation as $A=2\left(\lambda_{3}-\lambda_{2}\right)$. For an exact $N$-soliton solution of the $\mathrm{KdV}$ equation, with discrete eigenvalues at $\lambda=\lambda_{n}$ for $n=1, \ldots, N$ and continuous spectrum $\left(\lambda_{c}, \infty\right)$, the amplitude of each soliton would simply be $A_{n}=2\left(\lambda_{c}-\lambda_{n}\right)$. (Note that the point $\lambda_{c}$ not only marks the beginning of the continuous spetrum, but is also the constant value of the background on top of which the solitons propagate.) For general multi-phase solutions (i.e., genus larger than 1), however, the situation is more complicated. Since $\lambda_{2 n} \leqslant \mu_{n}(x, t) \leqslant \lambda_{2 n+1}$, one still has that the maximum amplitude of the $n$-th nonlinear excitation is $2\left(\lambda_{2 n+1}-\lambda_{2 n}\right)$. When considering the solitonic limit, however, there is no explicit formula that relates the amplitude of the solitons to the value of the points $\lambda_{n}$. As in past works, we will therefore use empirical estimates. This is perhaps the only point for which the available theory is not entirely satisfactory.

To deal with this complication, Osborne and Bergamasco introduced an arbitrary "reference" point $\lambda_{\text {ref }}$ for the spectrum, given by $\lambda_{\text {ref }}=\lambda_{2 J+1}$, where $J$ is the total number of solitons. Osborne and Bergamasco then identified the amplitude of the $n$-th soliton as

$$
A_{n, \mathrm{OB}}=2\left(\lambda_{\text {ref }}-\lambda_{2 n}\right) \text {. }
$$

As we will see shortly, however, Osborne and Bergamasco's predictions for the soliton amplitudes deviate from the values obtained numerically. Note that the reference level depends on number of solitons and, therefore, on the artificial threshold $\kappa$, which is less than ideal. Nonetheless, since no simpler or better or more justifiable expression is available at present, we will use (4.8) to estimate the soliton amplitudes.

As $\epsilon \rightarrow 0$, the band widths become exceedingly small, and it becomes reasonable to replace $\lambda_{2 n}$ by $z_{n}$, where as in section 2.3.3 $z_{n}$ is the $n$-th zero of the trace function. Substituting either of (3.5) into (4.8), we can write a fully explicit expression for the soliton amplitude by OB's method. For example, using the quadratic approximation for $z_{n}$ one obtains

$$
A_{n, \text { quadratic }}=2\left(\sqrt{8\left(2 N_{s}+1\right) \epsilon+32}-\sqrt{8(2 n-1) \epsilon+32}\right),
$$

and an even simpler expression is obtained using the linear approximation. In the following section we will compare these predictions to the results obtained from numerical simulations of the KdV equation. 


\section{Comparison with numerical experiments}

In the previous sections we have seen how the WKB method allows one to reconstruct the spectrum of the scattering problem for the $\mathrm{KdV}$ equation with cosine potential in the small dispersion limit. We now want to compare the results obtained from WKB to those obtained from numerical simulations of the KdV equation, including the ZK experiment.

Before we do so, we first validate the WKB results by comparing them to the results obtained by numerically computing the spectrum of the scattering problem. To obtain the spectrum of the scattering problem numerically, one can first convert (2.1) into a first order system and then apply standard numerical methods [e.g., a fourth-order Rungekutta (RK) algorithm] to numerically integrate the resulting system of ordinary differential equations and compute the monodromy matrix. The comparison between the WKB result and the numerical result, shown in Fig. 5 for $\epsilon=0.169$, shows excellent agreement.

\subsection{Comparison with Zabusky and Kruskal's experiment}

We now compare the WKB results with the results from direct numerical simulations of the KdV equation with small dispersion. Recall that Zabusky and Kruskal performed numerical simulations of the KdV equation with small dispersion in the form

$$
v_{\tau}+v v_{\xi}+\delta^{2} v_{\xi \xi \xi}=0,
$$

with initial condition $v(x, 0)=\cos (\pi \xi)$ and $\delta=0.022$ [33]. It is straightforward to verify that the corresponding scattering problem is given by [19]

$$
\delta^{2} w_{\xi \xi}+\left(\lambda_{\mathrm{zk}}+\frac{v}{6}\right) w=0 .
$$

Equations (5.1) and (5.2) are slightly different from the corresponding ones that were used in the previous sections. This is not a significant complication, however, since one can trivially transform them into the standard KdV equation (1.1) and scattering problem (2.1). Explicitly, we simultaneously rescale equation (5.1) into (1.1) as well as equation (5.2) into (2.1) by performing the change of variables

$$
x=\pi \xi, \quad t=(\pi / 6) \tau, \quad \lambda=6 \lambda_{\mathrm{zk}}, \quad u(x, t)=v(\xi, \tau),
$$

which implies

$$
\epsilon=\sqrt{6} \pi \delta .
$$

Zabusky and Kruskal chose the value $\delta=0.022$ for their numerical simulations. Correspondingly we need to take $\epsilon=0.169$ in order to compare our predictions with their results.

We first discuss the number of solitons. Recall that ZK identified eight solitons in their numerical simulations. Based on the discussion in section 4.1, for $\epsilon=0.169$ and $\kappa=0.99$, we have $\lambda_{s}=0.69$ and $\lambda_{s, \text { approx }}=0.684$. For both choices, (4.6) yields $N_{\text {soliton }}=8$, which agrees perfectly with the ZK experiment. Note, however, that total the number of nonlinear excitations in the ZK experiment is larger than eight. Indeed, the WKB estimate obtained in section 3 yields $N_{\text {zeros }}=11$ for the $\mathrm{ZK}$ experiment. This prediction is corroborated by the traces visible in Fig. 1(right) and is further validated by a numerical computation of the trace function (as shown in Fig. 5). These further, non-solitonic nonlinear excitations were (perhaps misleadingly, in the sense specified in this paper) referred to "hidden" or "virtual" solitons" in [3,26]. They correspond to very shallow and generally wide excitations which become mostly visible through their interaction with the main nonlinear (i.e, soliton-like) waves, while they appear well separated from the latter only for some rare, brief ranges of time.

Next we compare the soliton amplitudes obtained numerically from the ZK experiment with the values predicted by Osborne and Bergamasco's expression (4.8), using either the full WKB results or the quadratic approximation (4.9), as discussed in section 4.2. Note that $\lambda_{\text {ref }}=0.722$ for $\epsilon=0.169$. The ZK values can be extracted from Figure 1 in Ref. [33] or can equivalently be recovered by reproducing ZK's numerical simulations. The results are reported in Table 1 and illustrated in Figure 6. The maximum relative difference for the six largest solitons is $13 \%$ for the full WKB results and $12 \%$ for quadratic approximation (4.9). These results demonstrate very good agreement between the WKB predictions and the ZK experiment. Note, however, that the WKB predictions for the smaller solitons are not as precise. 
Table 1. Comparison of the soliton amplitudes from WKB to those from the ZK experiment

\begin{tabular}{ccccccccc}
\hline & 1 & 2 & 3 & 4 & 5 & 6 & 7 & 8 \\
\hline ZK & 3.019 & 2.678 & 2.340 & 1.991 & 1.610 & 1.212 & 0.805 & 0.463 \\
WKB + OB & 3.207 & 2.743 & 2.294 & 1.862 & 1.447 & 1.051 & 0.675 & 0.323 \\
WKB + quadratic & 3.205 & 2.746 & 2.304 & 1.877 & 1.464 & 1.064 & 0.674 & 0.295 \\
\hline
\end{tabular}

[Figures were moved to the end of the manuscript in compliance with submission instructions.]

Figure 6. Comparison of the soliton amplitudes from WKB to those from KdV simulations with $\epsilon=0.169$ (corresponding to the ZK experiment).

[Figures were moved to the end of the manuscript in compliance with submission instructions.]

Figure 7. The log-log plot of the number of solitons as a function of $\epsilon$. Red curve: full WKB predictions. Gray curve: WKB results with a linear expansion for $S_{2}(\lambda)$ and $S_{1}(\lambda)$. Black curve: WKB results with a linear expansion for $S_{2}(\lambda)$ and a quadratic expansion for $S_{1}(\lambda)$. Blue line: the number of nonlinear excitations from WKB. Orange circles: the number of solitons from full KdV numerics. Red circles: the number of nonlinear excitations from numerical solution of the scattering problem.

In their 1965 work [33], Zabusky and Kruskal also pointed out an interesting but unexplained property of the solitons, namely, the fact that the variation of the amplitude of the largest solitons is almost linear [33]. This phenomenon can easily be seen in Fig. 1 (left). This property, which had not been explained analytically to the best of our knowledge, can easily be explained using our asymptotic analysis of the spectrum. Specifically, recall that the $n$-th soliton amplitude is given by (4.9) and that, in the limit $\epsilon \rightarrow 0$, the quantity $\lambda_{2 n}$ can be replaced by $z_{n}$, given by (3.2). As illustrated in the left of Fig. 4, however, the function $S_{1}(\lambda)$ is almost linear near $\lambda=-1$. It is this linearity of $S_{1}(\lambda)$ near $\lambda=-1$ that leads to the linear distribution of the first zeros and eventually to the linear variation of the largest soliton amplitudes.

\subsection{Comparison with numerical simulations of the KdV equation in the small dispersion limit}

We now investigate the robustness of the WKB results as a function of $\epsilon$. Obviously, one expects that the WKB predictions will show better and better agreement with the results of numerical simulations as $\epsilon$ are taken to be progressively smaller. The question that we want to address here, however, is the opposite, namely: how large must $\epsilon$ become in order for the WKB results to lose their validity? The reason why this question is of interest is that, to the best of our knowledge, a comparison between the $\mathrm{KdV}$ results and real experiments in one-dimensional shallow water tanks was only carried out with relatively large values of $\epsilon$, which give rise to very few solitons [32]. It is therefore of interest to assess whether the asymptotic theory could be extended to quantitatively describe experiments carried out in regimes in which few solitons are present.

In Fig. 7 we plot the number of solitons as a function of $\epsilon$. The red curve shows the full WKB predictions. These are obtained from (4.6) by computing numerically the threshold value $\lambda_{s}$ and then evaluating $S_{1}\left(\lambda_{s}\right)$, as discussed in section 3. The gray curve shows the approximation obtained by considering the linear expansion for $S_{2}(\lambda)$ and $S_{1}(\lambda)$. The black curve shows the approximation obtained by considering the linear expansion for $S_{2}(\lambda)$ and the quadratic expansion for $S_{1}(\lambda)$. For comparison purposes, the blue line shows the total number of nonlinear excitations. The orange circles show the results obtained from the full KdV numerics, while the red circles show the results from numerical integration of the scattering problem. The comparison shows excellent agreement between the WKB predictions and the numerical results over a wide range of values of $\epsilon$.

Importantly, one should note that a similar problem to the one that arises in the asymptotics (namely, the need to introduce an arbitrary decision threshold in order to label an excitation solitonic or not) also appears in direct numerical simulations of the KdV equation. Namely: How does one extract the number of solitons from the numerical simulations? Or, in other words: How does one distinguish numerically between an effective soliton and a generic nonlinear excitation? This is a tricky problem because the resulting number can clearly depend on the method used. [E.g., using visual estimates in the ZK experiment, one would count eight solitons in Fig. 1(left), but in Fig. 1(right) 
there are at least ten excitations that are clearly emerging from the initial condition. and as many as fourteen excitations are visible at certain values of time; see section 7 for further discussion.] Note also that the scattering problem (1.2) and the $\mathrm{KdV}$ equation (1.1) itself become stiffer and stiffer as $\epsilon \rightarrow 0$, which makes the numerical simulations progressively more sensitive and difficult (and therefore potentially less reliable) in this limit.

\section{Arbitrary single-lobe periodic initial conditions}

In this section we show how the approach that we used for the Schrödinger equation (1.2) with cosine initial condition [discussed in section 2] can be generalized to analyze any continuous, single-lobe, periodic initial condition. More precisely, for the purposes of the present work we will call "single-lobe" any real-valued, periodic function with a single local maximum and a single local minimum within a period.

Given such a continuous, single-lobe initial condition $u(x)$ with period $L$, we denote respectively by $u_{\max }$ and $u_{\text {min }}$ its maximum and minimum values. The analysis of the spectrum of the Schrödinger operator with such a potential using the WKB method follows very closely the steps taken for the cosine initial condition. Namely, it is straightforward to see that, as before, one must study separately three different ranges of $\lambda$ : (i) $\lambda<u_{\min }$, (ii) $u_{\min }<$ $\lambda<u_{\max }$ and (iii) $\lambda>u_{\max }$. In each range, chosen an arbitrary point $x=x_{0}$, one first constructs a WKB approximation for a fundamental matrix solution over the interval $\left[x_{0}, x_{0}+L\right]$ and then evaluates the fundamental matrix solution at $x=x_{0}$ and $x=x_{0}+L$. In this way one can construct the monodromy matrix and therefore the trace function. Let us discuss the three ranges separately, like we did for the cosine initial condition.

For all $\lambda$ in range (i), following similar steps as in the case of the cosine initial condition, one finds that the trace of the monodromy matrix is given by

where now

$$
\operatorname{tr} M=2 \cosh \left(\tilde{S}_{\mathrm{i}} / \epsilon\right)
$$

$$
\tilde{S}_{\mathrm{i}}(\lambda)=\int_{x_{0}}^{x_{0}+L} \sqrt{u(x)-\lambda} \mathrm{d} x
$$

As in the case of the cosine initial condition, $\operatorname{tr} M>2$ for all $\lambda<u_{\min }$. [Indeed, $\tilde{S}_{\mathrm{i}}(\lambda)$ in $(6.2)$ reduces to $S_{\mathrm{i}}(\lambda)$ in (2.12) in the case of a cosine initial condition.] Hence all values $\lambda<u_{\text {min }}$ are not part of the spectrum of the scattering problem. Similarly, for all $\lambda$ in range (iii), following the same steps as before, one finds that the trace of the monodromy matrix is given by

where now

$$
\operatorname{tr} M=2 \cos \left(\tilde{S}_{\mathrm{iii}} / \epsilon\right)
$$

$$
\tilde{S}_{\mathrm{iii}}(\lambda)=\int_{x_{0}}^{x_{0}+L} \sqrt{\lambda-u(x)} \mathrm{d} x
$$

Note that, as in the case of the cosine potential, $|\operatorname{tr} M| \leqslant 2$ [And $\tilde{S}_{\mathrm{i}}(\lambda)$ in $(6.4)$ reduces to $S_{\mathrm{i}}(\lambda)$ in $(2.16)$ for a cosine initial condition.] Therefore all values of $\lambda$ in this range belong to the spectrum.

For range (ii), as in the case of the cosine potential we have two turning points $x_{1}$ and $x_{2}$ such that $u\left(x_{1}\right)=$ $u\left(x_{2}\right)=\lambda$ in the fundamental period $\left[x_{0}, x_{0}+L\right]$, which we take to be defined such that $x_{1}<x_{2}$. Without loss of of

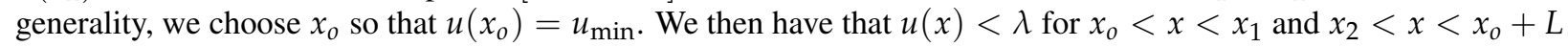
(or equivalently in the interval $x_{2}<x<x_{1}+L$ ), while $u(x)>\lambda$ for $x_{1}<x<x_{2}$. One can then perform similar calculations as in section 2 (including the expansions near the turning points and the computation of the connection formulae) to show that the trace of the monodromy matrix for all $\lambda$ in this range is given by

where now

$$
\operatorname{tr} M=2 \cosh \left[\tilde{S}_{2, \epsilon}(\lambda) / \epsilon\right] \cos \left(\tilde{S}_{1}(\lambda) / \epsilon\right),
$$

$$
\tilde{S}_{1}(\lambda)=\int_{x_{2}}^{x_{1}+L} \sqrt{\lambda-u(x)} \mathrm{d} x \quad \tilde{S}_{2}(\lambda)=\int_{x_{1}}^{x_{2}} \sqrt{u(x)-\lambda} \mathrm{d} x
$$


and $\tilde{S}_{2, \epsilon}(\lambda)=\tilde{S}_{2}(\lambda)+\epsilon \log 2$. Therefore, similarly to the case of the cosine initial condition, the range $\lambda \epsilon$ $\left[u_{\min }, u_{\max }\right]$ divides into bands and gaps, separated by a sequence of values $\lambda_{n}$ such that $\operatorname{tr} M\left(\lambda_{n}\right)= \pm 2$. [Note that, while $\tilde{S}_{2}(\lambda)=S_{2}(\lambda)$, we have $\tilde{S}_{1}(\lambda)=2 S_{1}(\lambda)$ in the specific case of a cosine initial condition. Correspondingly, the arguments of the cosine in (2.6) and (6.5) differ by a factor 2.]

As in the case of the cosine initial condition, the oscillations of the trace become more and more rapid in the small dispersion limit. Therefore, in this limit the band edges $\lambda_{n}$ can be approximated by the zeroes of $\cos \left(\tilde{S}_{1}(\lambda) / \epsilon\right)$. Omitting the calculations for brevity, one then finds that the $n$-th band width and relative band width are given by

$$
\begin{gathered}
w_{n}=\frac{2 \epsilon}{\left|\tilde{S}_{1}^{\prime}\left(\lambda_{n}\right)\right|} \operatorname{sech}\left[\tilde{S}_{2}\left(\lambda_{n}\right) / \epsilon\right]+O\left(\epsilon \mathrm{e}^{-2 \tilde{S}_{2}\left(\lambda_{n}\right) / \epsilon}\right), \\
W_{n}=\frac{4}{\pi} \operatorname{sech}\left[\tilde{S}_{2}\left(\lambda_{n}\right) / \epsilon\right]+O\left(\epsilon \mathrm{e}^{-\tilde{S}_{2}\left(\lambda_{n}\right) / \epsilon}\right) .
\end{gathered}
$$

One can now use these expressions to perform an asymptotic analysis of the spectrum to find essentially the same conclusions as in the case of a cosine initial condition.

\section{Concluding remarks}

In conclusion, we have shown how the judicious use of the WKB method in the scattering problem for the KdV equation with cosine initial data allows one to completely characterize the ZK experiments from an analytical point of view, including expressions for the total number of nonlinear excitations, the number of effective solitons and their amplitude, as well as providing an explanation for the approximately linear variation of the soliton amplitudes. Paraphrasing Scott Russell's comments after his observation of the "great wave of translation" (i.e., the solitary wave solution of the KdV equation): "It now remains for the mathematician to 'predict' the phenomenon after it has been discovered" [23]. The development of an analytical characterization of the solitary waves of the KdV equation took approximately fifty years until the work of Korteweg and de Vries [10]. Surprisingly, to the best of our knowledge it appears that a similarly long time has also elapsed to develop an analytical characterization of the ZK experiment.

We should reiterate the difference between nonlinear excitations and effective solitons, which is related to the presence of the so-called "hidden" solitons. Note in this respect that whether a given excitation gets to be called an effective soliton in any specific setting depends on the arbitrary choice of the threshold $\kappa$. This is of course less than ideal. At the same time, however, one should note that the specific value chosen for the threshold becomes less and less important in the limit $\epsilon \rightarrow 0$. This can easily be seen by noting that in the expressions (4.7) for the total number $N_{s}$ of effective solitons, the value of $\mathcal{K}$ only determines a constant offset, whereas the leading order, $\epsilon$-dependent contribution is independent of $\mathcal{K}$, and the $\kappa$-dependent terms become progressively smaller compared to the leading-order term as $\epsilon \rightarrow 0$.

A related issue is that of obtaining a precise count of the total number of nonlinear excitations. On one hand, as many as 14 excitations are visible for certain values of time in the contour plot in Fig. 1, as mentioned in section 5. On the other hand, looking at the solution profile as a function of $x$, at no time one can detect more than 10 local maxima. The same issue arises in the theoretical analysis of the spectrum, since on one hand the WKB analysis only predicts that 11 excitations are present in the ZK simulation, but on the other hand numerical simulations of the scattering problem clearly reveal three tiny gaps for $\lambda>1$. Such gaps will never be picked up by the WKB analysis discussed in this work, which (as discussed in section 2) predicts an infinitely long stable band for $\lambda>1$. But this discrepancy should not be surprising, because WKB provides an asymptotic characterization of the spectrum that is valid as $\epsilon \rightarrow 0$, and therefore it is to be expected that small discrepancies will exist for finite values of $\epsilon$.

The results of this work open up a number of interesting questions, from either a mathematical point of view or an applied one. For example:

1. A technical issue arising in the analysis is that the WKB approximation for the spectrum of the scattering problem is non-uniform for $\lambda \in \mathbb{R}$. More precisely, the WKB approximation for $\operatorname{tr} M$ has a jump discontinuity at $\lambda=-1$ and $\lambda=1$. This can easily be seen by comparing the values of (2.13) and (2.47) at $\lambda=-1$ and those of (2.17) and (2.47) at $\lambda=1$, respectively. In particular, $\lim _{\lambda \rightarrow 1^{+}} \operatorname{tr} M=2$, whereas $\lim _{\lambda \rightarrow 1^{-}} \operatorname{tr} M=5 / 2$, independently of $\epsilon$. Thus, the WKB approximation does not converge uniformly in any open subset of the real axis that 
includes the points $\lambda= \pm 1$. Whether this deficiency can be overcome by considering a higher-order approximation remains a problem left for further investigation.

2. A more general open problem is whether one can obtain a more detailed characterization of the dispersionless limit for arbitrary (i.e., multi-hump) periodic potentials beyond the results of [28]. Note that, from the point of view of WKB, treating the case of arbitrary potentials becomes quickly impractical due to the presence of multiple turning points. A related difficulty here is that, even though many results exist on the use of the IST to study the dispersionless limit of the KdV on the infinite line with decaying initial data (e.g., see $[4,7,12,13,27]$ and references therein), the IST machineries to solve the IVP in the infinite line case and the periodic case are very different, and although one can formally take the infinite period limit of the latter [29], such a limit is highly nontrivial, and it is not clear that it can be used to characterize the dispersionless limit.

3. From a more practical point of view, an open question is whether it is possible to derive Osborne and Bergamasco's expression for the soliton amplitudes from first principles, and whether a different expression can be obtained that yields better agreement with the numerical results.

4. Finally, it remains a very interesting open question whether it is possible to perform a comparison between the ZK results, the WKB predictions and real, experimental data in water waves. In this respect we note that, even though the breakup of an initial profile has been observed in experiments [32], fifty years after ZK the soliton recurrence still has not been experimentally observed to the best of our knowledge.

We hope that the above questions will stimulate further research on these and related problems in the near future.

\section{Acknowledgments}

We thank M. Onorato for many insightful discussions on issues related to the present work. This work was partially supported by the National Science Foundation under grant number DMS-1311847. S.T. acknowledges funding from Italian Ministry of the University under grant PRIN 2012BFNWZ2.

\section{References}

\section{References}

[1] M. J. Ablowitz and H. Segur, Solitons and the inverse scattering transform, SIAM (1981)

[2] C. M. Bender and S. A. Orszag, Advanced mathematical methods for scientists and engineers, Springer (1978)

[3] I. C. Christov, Hidden solitons in the Zabusky-Kruskal experiment: analysis using the periodic, inverse scattering transform, Mathematics and Computers in Simulation 82, (2012), pp. 1069-1078

[4] P. Deift, S. Venakides and X. Zhou, An extension of the steepest descent method for Riemann-Hilbert problems: the small dispersion limit of the Korteweg-de Vries (KdV) equation, Proc. Nat. Acad. Sci. USA 95 (1998), 450-454.

[5] B. A. Dubrovin and S. P. Novikov, Periodic and conditionally periodic analogs of the many-soliton solutions of the Kortewegde Vries equation, Sov. Phys. JETP. 40 (1974) 1058-1063

[6] C. S. Gardner, J. M. Greene, M. D. Kruskal, R. M. Miura, Method for solving the Korteweg-deVries equation, Phys. Rev. Lett. 19 (1967) 1095-1097

[7] T. Grava and F. R. Tian, The generation, propagation, and extinction of multiphases in the KdV zero-dispersion limit, Commun. Pure Appl. Math. 55 (2002) 1569-1639.

[8] P. Hartman, Ordinary differential equations, SIAM (1982)

[9] B. Herbst, G. Nieddu and A. D. Trubatch, Recurrence in the Korteweg-deVries equation?, Contemp. Math. 635 (2015) 1-12

[10] D. Korteweg and G. deVries, On the Change of Form of Long Waves Advancing in a Rectangular Canal, and on a New Type of Long Stationary Waves, Philosophical Magazine Ser.5, 39 (1895) 422-443

[11] P. D. Lax, Periodic solutions of the KdV equation, Communications on pure and applied mathematics 28, pp. 141-188 (1975),

[12] P. D. Lax and C. D. Levermore, The small dispersion limit of the Korteweg-de Vries equation. I. II. III., Comm. Pure Appl. Math. 36, pp. 253-290; 571-593; 809-829 (1983).

[13] P. D. Lax, C. D. Levermore, and S. Venakides, The generation and propagation of oscillations in dispersive initial value problems and their limiting behavior, Important developments in soliton theory, 205-241. Springer Series in Nonlinear Dynamics. Springer, Berlin (1993).

[14] W. Magnus and S. Winkler, Hill's equation, John Wiley and Sons, Inc. (1966)

[15] A. Messiah, Quantum mechanics, Elsevier (1961)

[16] S. P. Novikov, The periodic problem for the Korteweg-deVries equation, Funct. Anal. Appl. 8 (1974) 236-246 
[17] S. Novikov, S. V. Manakov, L. P. Pitaevskii, V. E. Zakharov, Theory of solitons: the inverse scattering method, Springer (1984)

[18] F. W. J. Olver, D. W. Lozier, R. F. Boisvert, C. W. Clark, Eds. NIST Handbook of Mathematical Functions, Cambridge University press (2010)

[19] A.R. Osborne and L. Bergamasco, The solitons of Zabusky and Kruscal revisted: perspective in terms of the periodic spectral transform, Physica 18D (1986) 26-46

[20] A. R. Osborne and M. Petti, Laboratory-generated, shallow-water surface waves: analysis using the periodic, inverse scattering transform, Phys. Fluids 6 (1994) 1727-1744

[21] A. R. Osborne, E. Segre, G. Boffetta, L. Cavaleri, Soliton basis states in shallow-water ocean surface waves, Phys. Rev. Lett. 67 (1991) 592-595

[22] M. J. Richardson, Solution of the Mathieu equation in the WKB approximation, American Journal of Physics 39 (1970) $560-565$

[23] J. S. Russell, Report of the committee on Waves, Report of the seventh meeting of the British Association for the Advancement of Science, Liverpool, (1838) 417-496

[24] A. Salupere, G. A. Maugin, and J. Engelbrecht, Korteweg-de Vries soliton detection from a harmonic input, Phys. Lett. A 192 (1994) 5-8

[25] A. Salupere, G. A. Maugin, J. Engelbrecht, and J. Kalda, On the KdV soliton formation and discrete spectral analysis, Wave Motion 23 (1996) 49-66

[26] A. Salupere, J. Engelbrecht, and P. Peterson, On the long-time behaviour of soliton ensembles, Math. Comp. Simul. 62 (2003) $137-147$

[27] S. Venakides, The zero dispersion limit of the Korteweg-de Vries equation for initial potentials with nontrivial reflection coefficient, Comm. Pure Appl. Math. 38 (1985)125-155

[28] S. Venakides, The zero dispersion limit of the Korteweg-deVries equation with periodic initial data, Trans. Amer. Math. Soc. 301 (1987) 189-226

[29] S. Venakides, The infinite period limit of the inverse formalism for periodic potentials, Commun. Pure Appl. Math. 41 (1988) 3-17

[30] N. J. Zabusky, Nonlinear lattice dynamics and energy sharing, J. Phys. Soc. Japan 26 Suppl., 196 (1969)

[31] N. J. Zabusky Computational synergetics and mathematical innovation, J. Comput. Phys. 43 (1981) 195-249

[32] N. J. Zabusky and C. J. Galvin, Shallow-water waves, the Korteweg-deVries equation and solitons, J. Fluid Mech. 47 (1971) 811-824

[33] N. J. Zabusky and M. D. Kruskal, Interaction of solitons in a collisionless plasma and the recurrence of initial states, Phys. Rev. Lett. 15 (1965) 240-243 

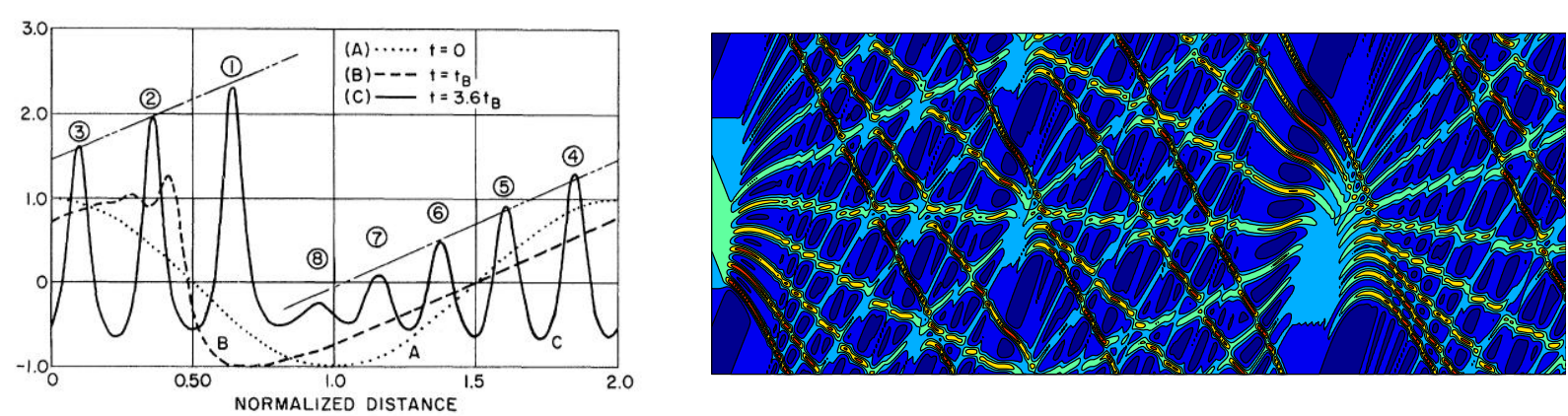

Figure 1. Left: The eight solitons emerging from the Zabusky-Kruskal experiment; from Ref. [33]. Right: Contour plot of the solution of the Zabusky-Kruskal experiment. The vertical axis is space, and the horizontal axis is time. Note how only eight peaks are visible in the left figure, whereas at least ten excitations can be clearly identified in the right figure.

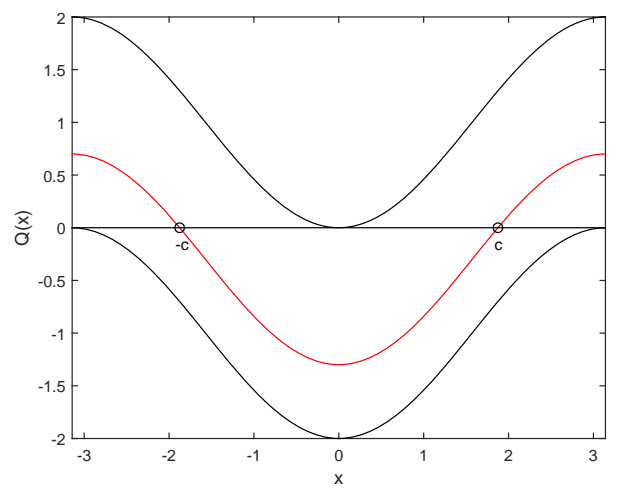

Figure 2. $Q(x)=\lambda-\cos x$ as a function of $x$ over $[-\pi, \pi]$ for $\lambda= \pm 1$ (black curves) and $\lambda=-0.3$ (red curve).

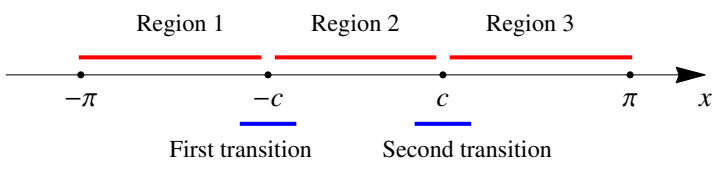

Figure 3. The various sub-regions of the fundamental domain $x \in[-\pi, \pi]$ for the WKB analysis in the range $-1<\lambda<1$.
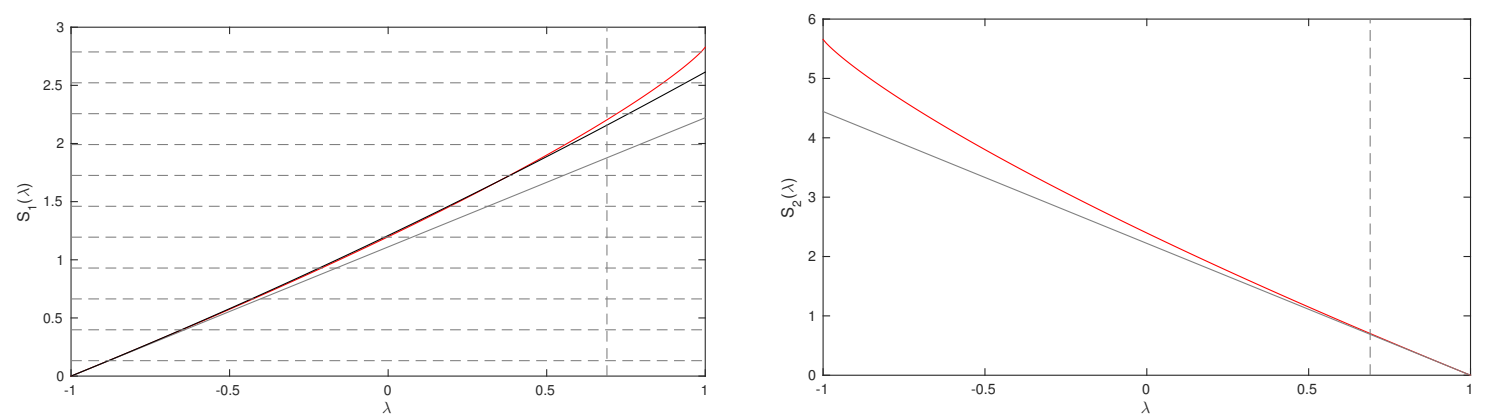

Figure 4. Left: $S_{1}(\lambda)$ as a function of $\lambda$ (red curve). Right: $S_{2}(\lambda)$ as a function of $\lambda$ (red curve). The solid gray curves show the linear approximation of $S_{1}(\lambda)$ and $S_{2}(\lambda)$ about $\lambda=-1$ and $\lambda=1$, respectively. The black curve shows the second-order Taylor expansion of $S_{1}(\lambda)$ about $\lambda=-1$. The dashed gray horizontal lines show the levels $S_{1}(\lambda)=\frac{1}{2}\left(n-\frac{1}{2}\right) \pi \epsilon$, where $n=1,2 \ldots$, for $\epsilon=0.169$ (corresponding to the ZK experiment; cf. section 5 for details). The dashed vertical line shows the threshold value $S_{2}(\lambda)=\epsilon \ln \left(\frac{2}{\pi \kappa}\right)$, where $\kappa=0.01$. 


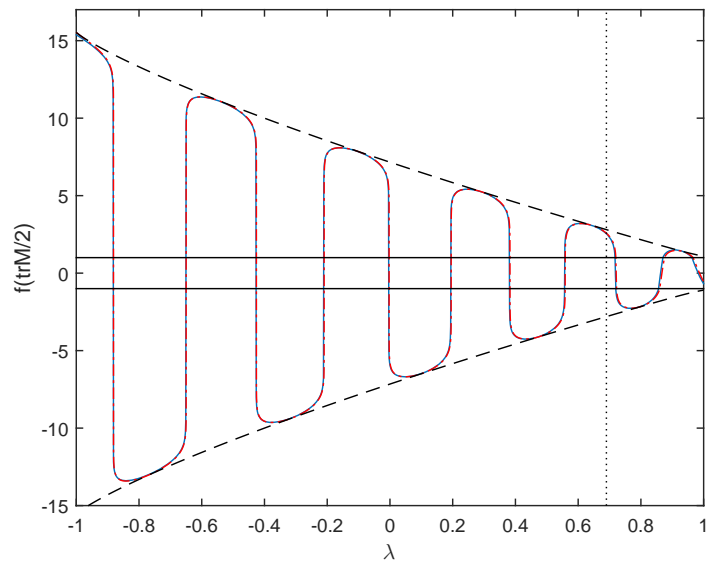

Figure 5. The function $f(\operatorname{tr} M / 2)$ defined by (3.1) as a function of $\lambda \in[-1,1]$ for $\epsilon=0.169$ (corresponding to the ZK experiment; cf. section 5 for details). Dash-dotted red curve: the expression (2.47) obtained via WKB. Solid blue curve: results from numerical integration of the scattering problem (cf. section 5 for details). (Note that the WKB results are almost indistinguishable from those from full numerics, and as a result the blue curve is hidden by the red curve.) Black curves (dashed): the envelope function $\pm \cosh \left[S_{2, \epsilon}(\lambda) / \epsilon\right]$ from (2.47). The dotted vertical line denotes the solitonic threshold $\lambda_{s}$ (cf. section 3 for details).

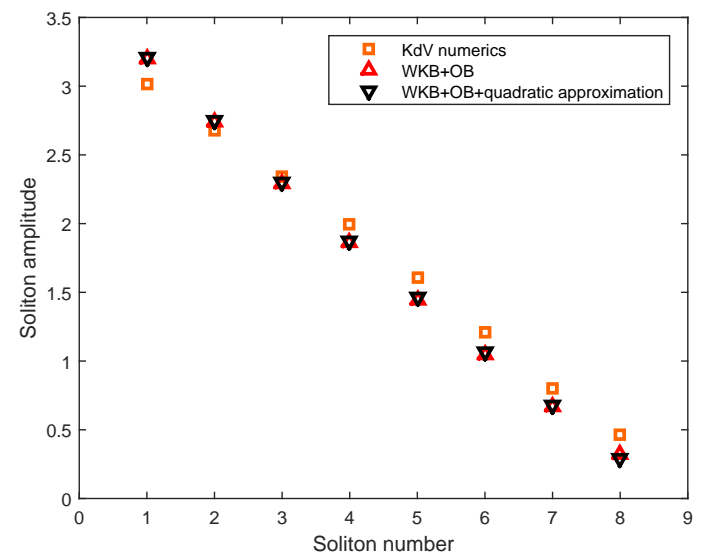

Figure 6. Comparison of the soliton amplitudes from WKB to those from KdV simulations with $\epsilon=0.169$ (corresponding to the ZK experiment).

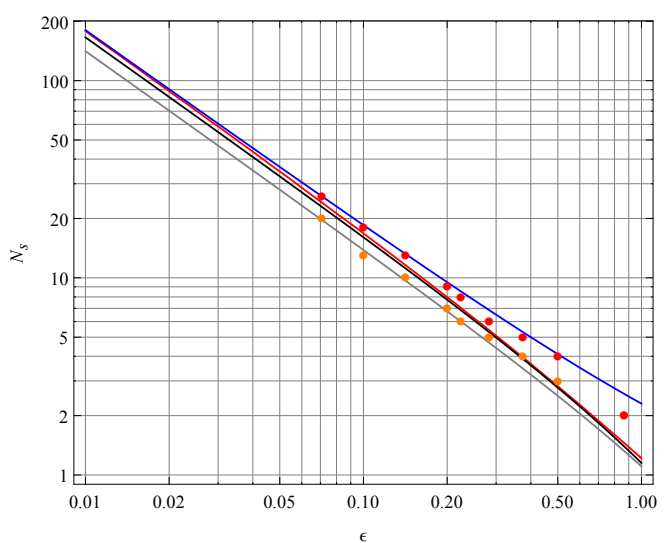

Figure 7. The log-log plot of the number of solitons as a function of $\epsilon$. Red curve: full WKB predictions. Gray curve: WKB results with a linear expansion for $S_{2}(\lambda)$ and $S_{1}(\lambda)$. Black curve: WKB results with a linear expansion for $S_{2}(\lambda)$ and a quadratic expansion for $S_{1}(\lambda)$. Blue line: the number of nonlinear excitations from WKB. Orange circles: the number of solitons from full KdV numerics. Red circles: the number of nonlinear excitations from numerical solution of the scattering problem. 